\title{
DESIGN AND IMPLEMENTATION OF AUTONOMOUS MANEUVERING ROBOTIC VEHICLE USING GLOBAL POSITIONING SYSTEM FOR JAMMING THE COMMUNICATION DURING WARFARE
}

\author{
V.Sumanth ${ }^{1}$, M. E. C. Vidya Sagar ${ }^{2}$, Sk. Nowshad ${ }^{3}$ \\ 1, 2, 3 Sr. Assistant professor, Dept of EIE, Narayana Engineering College-Nellore
}

\begin{abstract}
In this paper we present the construction of an autonomous self navigating robotic vehicle. This robotic vehicle uses GPS to automatically navigate itself to the instructed location. Once the robot reaches the desired location it turns on a RF Transmitter which is tuned to the communication frequency of the opposing unit. This RF transmitter transmits random noise continuously. Since the transmission is in the same frequency as that of the communication frequency of the opposing unit, their communication will become noisy and they will be unable to do any meaningful communication. The robotic vehicle essentially contains a GPS receiver, a microcontroller unit and a RF transceiver, in addition to the motors that run the robotic vehicle. The robotic vehicle can be commanded to go to a certain location using the GPS co-ordinates of the location. These co-ordinates are given to the robot using a $R F$ data link. Once the robot receives the GPS co-ordinates, the robot starts its process and automatically navigates itself to the commanded location with the help of the on board GPS receiver.
\end{abstract}

\section{FUNCTIONAL BLOCK DIAGRAMS OF THE}

\section{SYSTEM}

There are two main modules in the design of Robotic vehicle. They are

1. Transmitter module \&

2. Robotic vehicle module

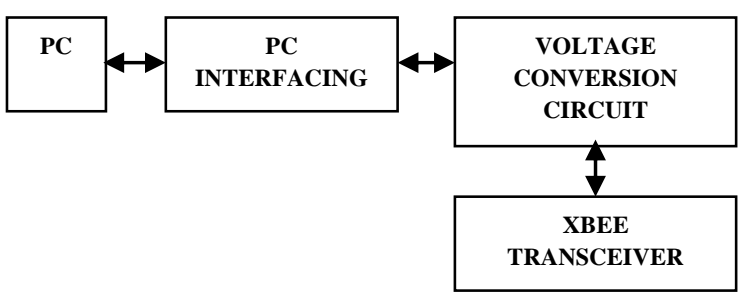

Fig. 1:Functional Block diagram of the Transmitter module

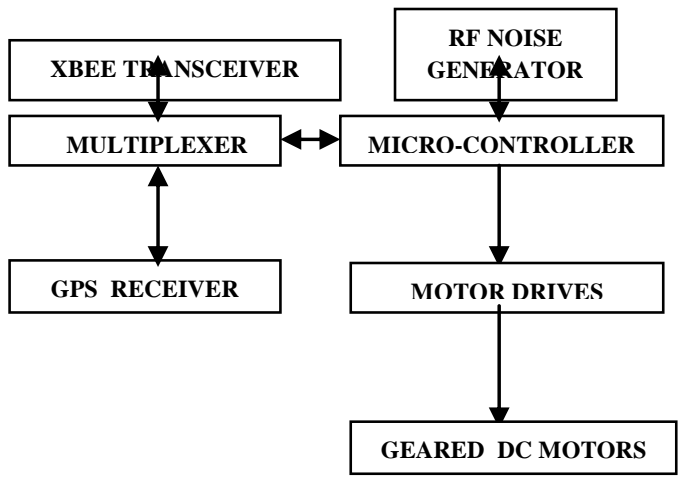

Fig. 2 : Functional Block diagram of Robotic vehicle module

\section{EXPLANATION}

Personal Computer is used to display the information of the Robotic Vehicle. The Interfacing Circuit is necessary for the communication or transferring data between two modules in transmitter module. The voltage levels of the PC interfacing circuit and $\mathrm{X}$ bee[2] transceiver are different from each other. The PC interfacing circuit works at $5 \mathrm{~V}$ and the $\mathrm{X}$ bee transceiver at $3.3 \mathrm{~V}$. In order to convert the voltage to the required levels the voltage conversion circuit is used. $\mathrm{X}$ bee transceiver is used for to and fro communication. It guides the actual position of the robotic vehicle to the Personal computer.

The GPS Receiver in the robotic vehicle module receives the co-ordinates in terms of latitudes and longitudes where the vehicle is present. The multiplexer is used to combine both the co-ordinates of GPS receiver and X bee transceiver. It is then given to microcontroller unit. Microcontroller unit is the heart of the system. The position of the Robotic vehicle and GPS co-ordinates are compared and the corresponding movement is given to the wheels of the Robotic vehicle. Geared DC Motor drives the robotic vehicle to a desired position if commanded by the controller. After reaching the target area, the relay will be $\mathrm{ON}$ and noise will be generated. 


\section{SCHEMATIC OF THE TRANSMITTING}

\section{MODULE AND ROBOTIC VEHICLE MODULE}

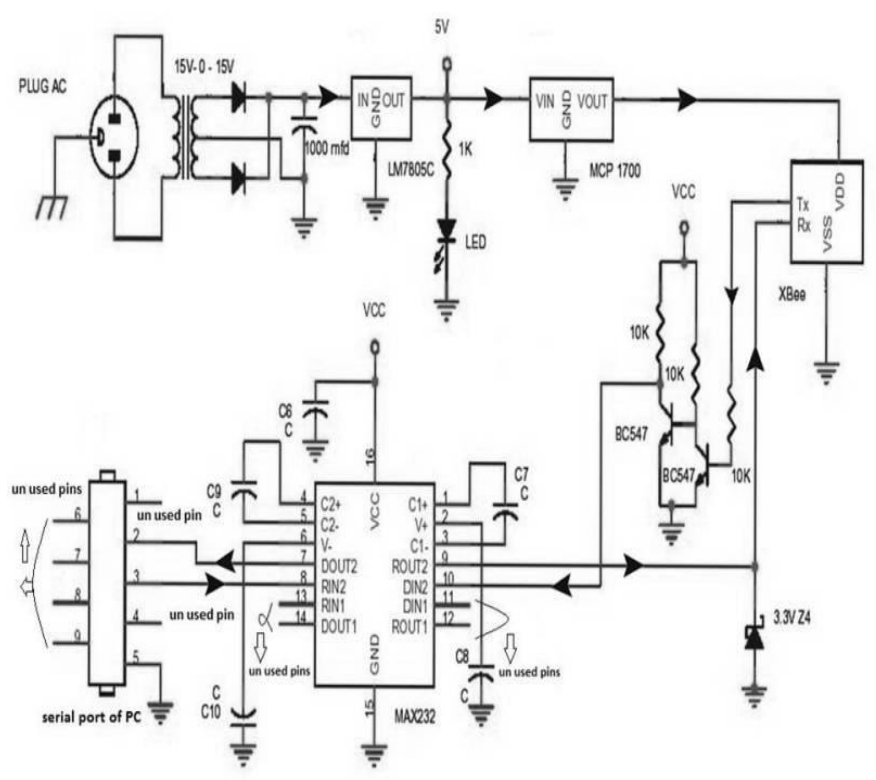

Fig.3: Schematic of Transmitter module

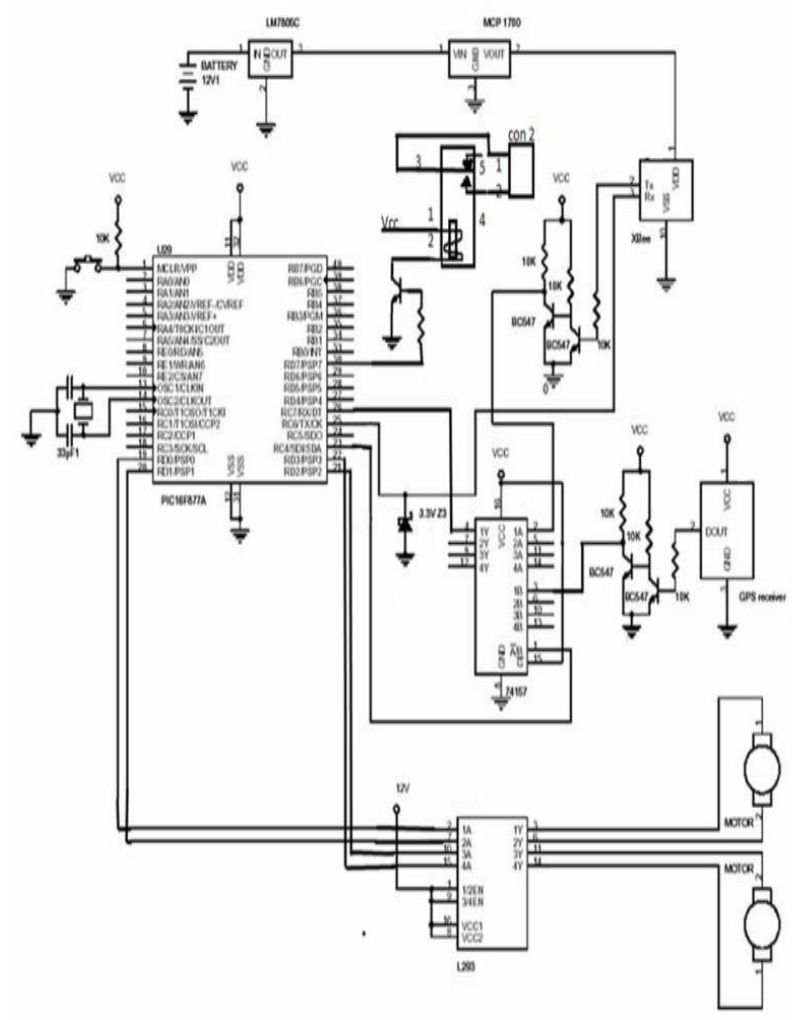

\section{INTERFACING \& IMPLEMENTATION OF THE SYSTEM}

The most common communication interface for short distance is RS-232. RS-232 defines a serial communication for one device to one computer communication port. The 9-pin connector is used as shown below.

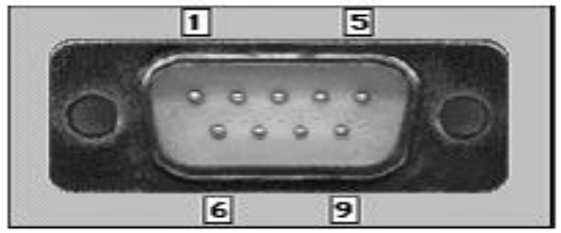

Fig.5: Recommended Standard 232 Cable

MAX 232 IC is used as an interface between personal computer and transmitter module. The MAX232 is a dual driver/receiver that includes a capacitive voltage generator to supply TIA/EIA-232-F voltage levels from a single 5-V supply. Each receiver converts TIA/EIA-232-F inputs to 5-V TTL/CMOS levels.

These receivers have a typical threshold of $1.3 \mathrm{~V}$, a typical hysteresis of $0.5 \mathrm{~V}$, and can accept \pm 30 - $\mathrm{V}$ inputs. Each driver converts TTL/CMOS input levels into TIA/EIA-232-F levels. $\mathrm{XBee}$ is one of the wireless protocols based on the IEEE 802.15.4 standard for wireless personal area networks (WPANs). XBee is designed to use in embedded applications requiring low data rates and low power consumption. The $\mathrm{XBee}$ module is as shown below.

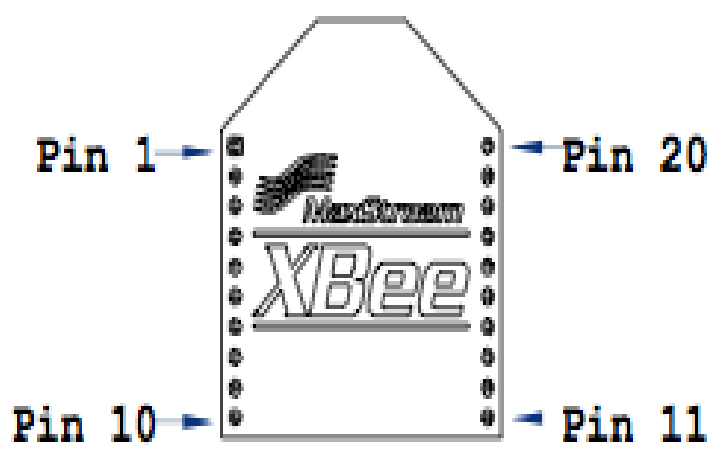

Fig.4: Schematic of Robotic vehicle module 


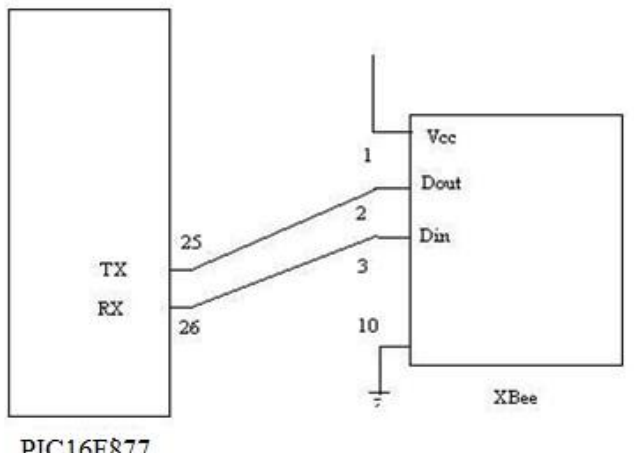

PIC16F 877

Fig. 7: Schematic of PIC to XBee connection

PIC16F877 is used as a main microcontroller of the project. Since PIC16F877 provides UART interface, transmitting data pin (TX) and the receiving data pin (RX) of the PIC will be directly connected to DIN and DOUT pins in the XBee module.Transmitted data will directly go to the DOUT pin and Received data from another module will go to the RX pin.

Programming is simple as the configuration.

\section{Get data from XBee:}

rxed = getcUSART();

//get a single character off the USART line (took out while(BusyUSART());

while(BusyUSART());

\section{Send data to XBee:}

putcUSART(' $\mathrm{C}$ '); //sending character $\mathrm{C}$ to the modules while(BusyUSART());

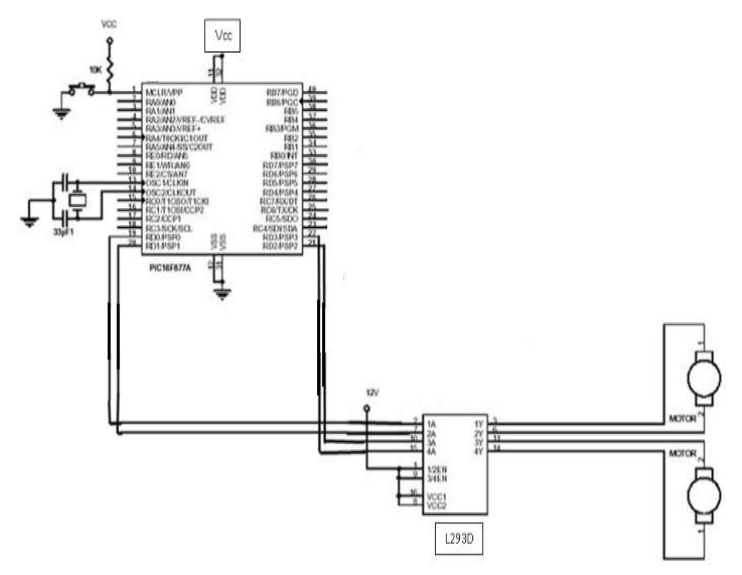

Fig.8:Schematic circuit for interfacing Motor IC with PIC Microcontroller
In order to drive the motor, motor drive IC is needed .Motor drive used in project is IC L293D.This IC helps to interface motor to circuit.The Pin diagram is as shown below

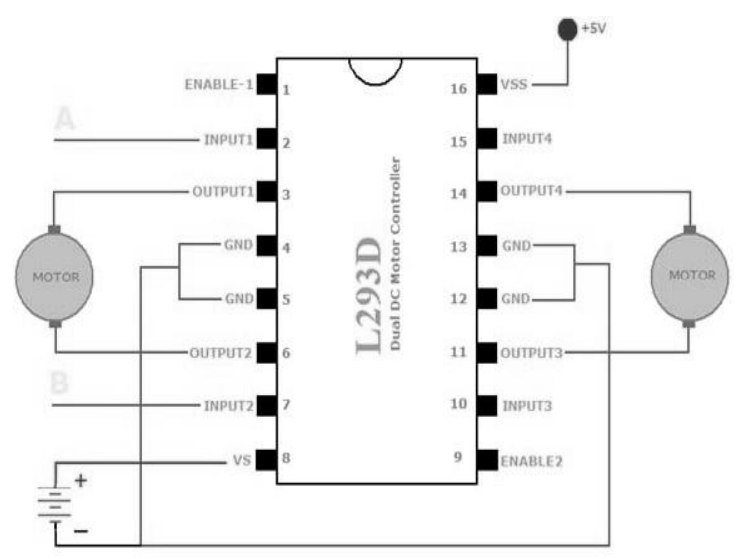

Fig.9: Pin diagram of IC L293D

\section{WORKING OF A H-BRIDGE FOR MOTOR CONTROL}

Usually a DC Motor like most DC devices has polarity and the direction of rotation of the motor changes with the polarity. For example in the two circuits shown below the polarity of the power supply to the motor is opposite and hence the direction of rotation of the motor will also be opposite in both the cases. In the circuit 1 if the motor rotates in the clockwise direction then in circuit 2 the motor will rotate in anticlockwise direction.
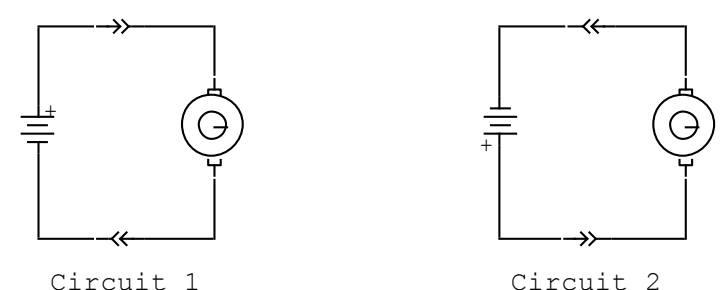

Fig.10: Motor working diagrams

Hence for making a DC motor run in opposite direction the polarity of the power applied to the motor must be changed or in other words the direction of the current through the motor must be changed.

In practical applications (for example robotic vehicles) the polarity cannot be interchanged manually and hence there is a need to form a circuit using which the direction of current through a motor can be changed. One such commonly used circuit configuration is a $\mathrm{H}$ Bridge. 


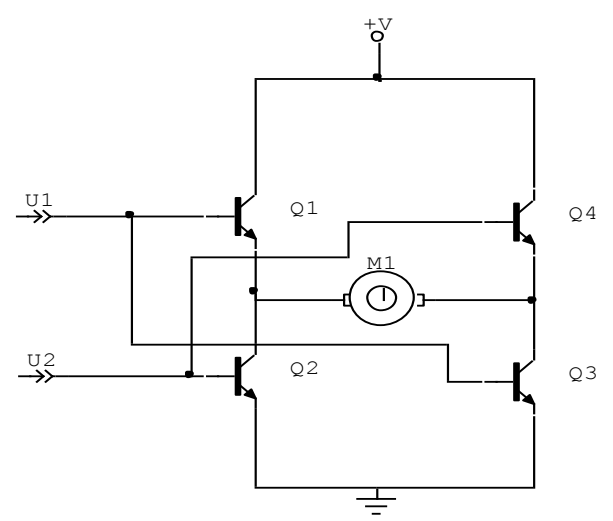

Fig.10: Circuit Diagram of H-Bridge

An $\mathrm{H}$ Bridge is a circuit that is constructed using four switching devices (transistors / FET/ IGBT). A transistor based $\mathrm{H}$ bridge is shown above. Using the above circuit the direction of current through the motor (M1) can be changed, by controlling the four transistors.

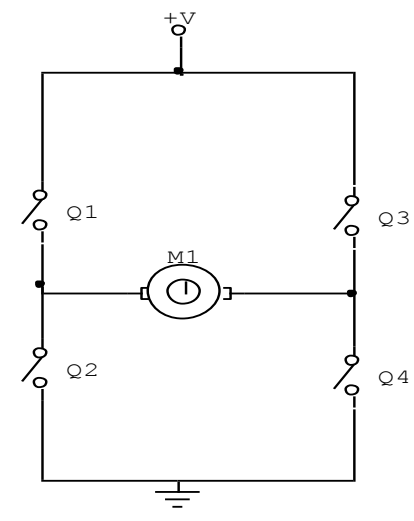

Fig.10.1: Working of H-Bridge

To understand the working of an $\mathrm{H}$ Bridge the transistors can be thought of as four switches connected as shown above. When the switches Q1 and Q4 are closed, the current through the motor flows in the direction, as shown below.

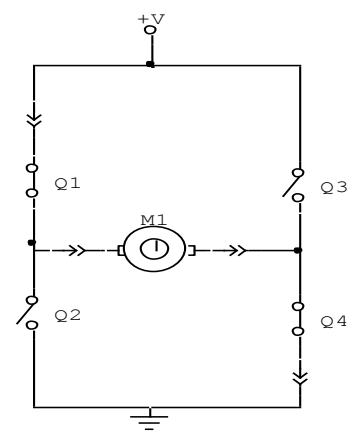

Fig.10.2: Working of H-Bridge
Similarly when the switches Q3 and Q2 are closed as shown below, the current flows in the opposite direction. Thus using a H Bridge the direction of current through a DC motor can be changed by controlling the four switching devices used in the bridge.

In the transistor circuit shown earlier the base of the Q1 and Q4 are connected together and similarly the base of the transistors Q2 and Q3 are also connected together. When a high signal is given to the input $\mathrm{U} 1$, transistors Q1 and Q4 turn on and thus allowing the current to flow through the motor in one direction. Alternately when the high signal is applied to $\mathrm{U} 2$, transistors Q2 and Q3 turn on, allowing the current to flow in the opposite direction. Thus four switching devices connected in a $\mathrm{H}$ bridge configuration can be used to make a motor rotate in opposite directions, as required in most robotic applications.

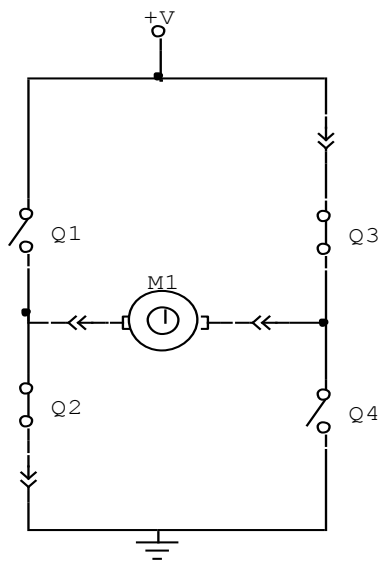

Fig.10.3: Working of H-Bridge

The $\mathrm{H}$ Bridge can either be constructed using four discrete transistors or alternately ICs like the L293D can be used. The IC L293D consists of four half ' $\mathrm{H}$ ' bridges, in a 16Pin IC package and using the four half ' $\mathrm{H}$ ' Bridges, two full bridges can be constructed.

\section{GLOBAL POSITIONING SYSTEM}

A network of satellites that continuously transmit coded information, which makes it possible to precisely identify locations on earth by measuring distance from the satellites. As stated in the definition above, GPS stands for Global Positioning System, and refers to a group of U.S. Department of Defense satellites constantly circling the earth. The satellites transmit very low power radio signals allowing anyone with a GPS receiver to determine their location on Earth. This remarkable system was not cheap to build, costing the U.S. billions of dollars. Ongoing maintenance, including the launch of replacement satellites, adds to the cost of the system. Amazingly, GPS actually predates the introduction of the personal computer. Its designers may not have foreseen a day when we would be carrying small portable receivers, weighing less than a pound, that would not only tell us where 
we are in position coordinates (latitude/longitude), but would even display our location on an electronic map along with cities, streets and more. These designers originally had military application in mind. GPS receivers would aid navigation, troop deployment and artillery fire (among other applications). Fortunately, an executive decree in the $1980 \mathrm{~s}$ made GPS available for civilian use also. Now everyone gets to enjoy the benefits of GPS! The capability is almost unlimited.

\section{WORKING OF GPS}

GPS relies on the principle of trilateration to find location. Specifically, there is a set of 29 satellites (last one launched in November, 2004) orbiting the Earth; at any one time, 24 are sending signals. The satellites are stationary 12,000 miles above the Earth so the same satellite passes you at the same time each day. Each satellite beams a unique "song" (really a set of low frequency vibrations called a "pseudo-random code") that radiates out spherically. GPS receiver listens to the incoming song and compares it to its own recorded version. Because the satellite is thousands of kilometers out in space, the two signals will be offset. The GPS unit corrects the offset and uses time-difference to calculate true distance. Each satellite contains an atomic clock and the GPS receiver sets its own internal clock to match the satellite. The signal from each satellite radiates out as a sphere, so the intersection of two spheres_is a circle. Therefore, with two satellites location can be found onto a large circle. By adding another satellite's signal, the intersection becomes two points. The GPS unit reads three satellites and relies on other variables to find the correct option between the two choices (2-D positioning). It is also possible to read the signal from four satellites to more accurately locate position (3-D positioning). The location of each satellite is monitored by a ground-based network and the information is beamed back to the satellites which, in turn, relay the information to the GPS receivers.

\section{GGM309R GPS RECEIVER}

Altina's GGM309R GPS Receiver provides various applications such as car navigation, marine navigation, mapping, surveying, security, agriculture and so on. It communicates with device (such as Pocket PC or notebook) via compatible dual-channel through RS-232 or TTL and saves satellite data by built-in backup memory. Low power consumption technology enables GGM309R and the device to save more operating power. Furthermore, GGM309R can track up to 20 satellites at a time, re-acquire satellite signals in $100 \mathrm{~ms}$ and update position data every second. Trickle-Power allows the unit to operate a fraction of the time, and Push toFix permits user to have a quicker position fix even though the receiver stays off.
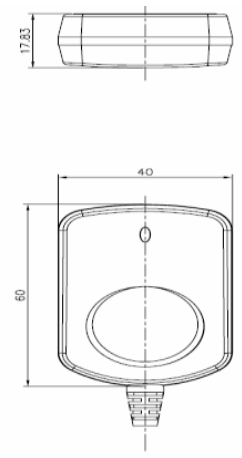

Fig.11: Hardware diagram of GGM30R GPS Receiver

\section{FLOW CHART}

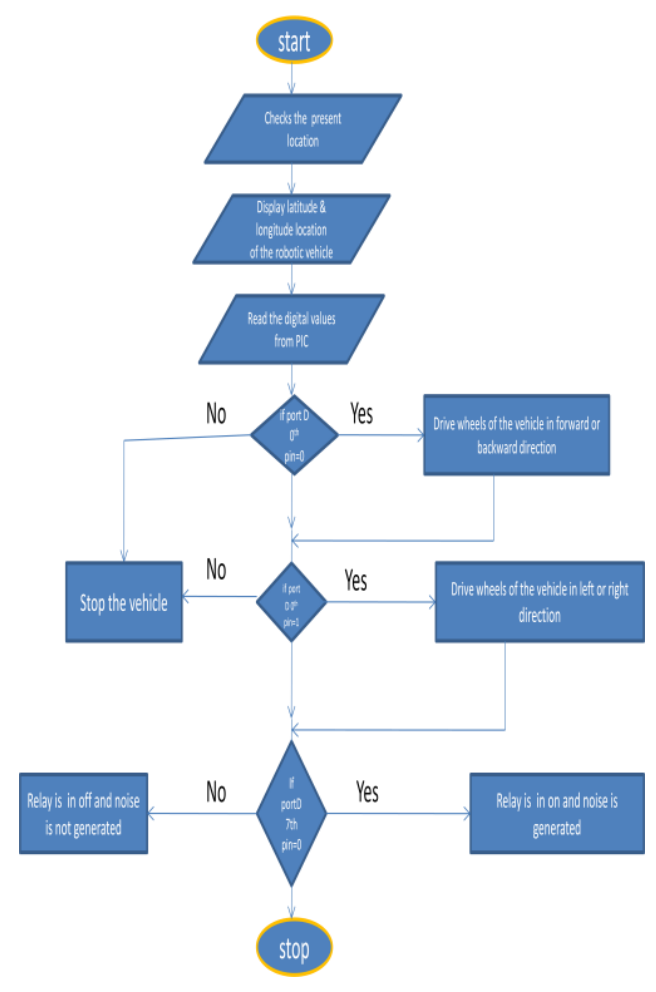

RESULT \& FUTURE SCOPE

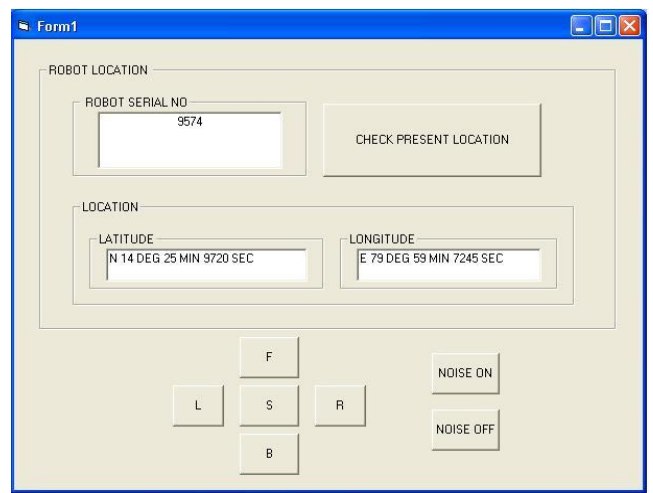


The above window is designed using Visual basic software and the program is dumped in the PIC controller using KEIL compiler.The window provides exact location the vehicle in terms of latitude and longitude and by pressing the radio buttons noise can added up with the communicating signal. The communication is performed using a pair of walky talky devices.

Army areas of interest for unmanned ground system technologies include the following capabilities.

- Supervised autonomous navigation that can maneuver through rough terrain under adverse environmental and lightening conditions.

- Unmanned reconnaissance, surveillance, target acquisition, scout and engagement.

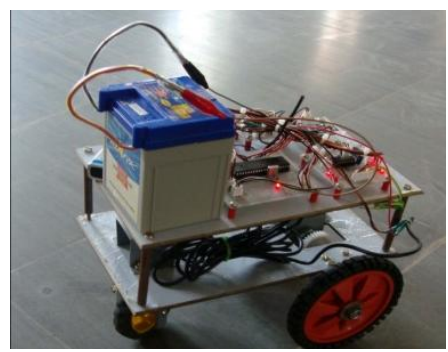

Fig.12: Robotic vehicle Module

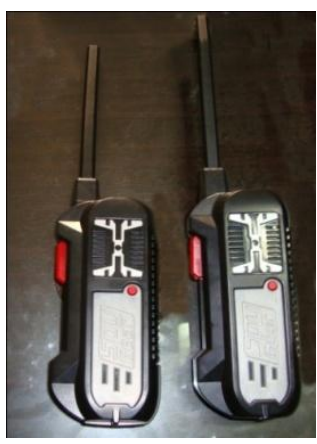

Fig.13: Walky Talky Devices

\section{REFERENCES}

[1].Smooth motion Planning for car like vehicles by F.Lamiraux and J.P.Laumond IEEE Transactions on Robotics \& Automation VOL.17,No.4,August 2001.

[2].Improved GPS sensor model for mobile robots in urban terrain, Department of Computer science, university of Freiburg, Germany.

[3].Microcontrollers Architecture, Programming, Interfacing and System Design by Rajkamal

[4].ZigBee Wireless Networks and Transceivers By Shahin Farahani and data sheets by maxstream.

[5].www.ti.com and data sheets by Texas Instruments

[6].www.microchip.com
[7].Industrial Robotics by Groover

\section{BIOGRAPHIES:}

V.SUMANTH received the M.E; degree in

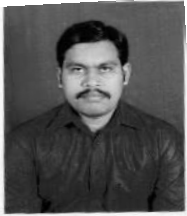

Electronics \& Control Engineering from Sathyabama University in 2011.Currently, he is working as Senior Assistant professor in the Department of Electronics \& Instrumentation Engineering at Narayana Engineering College, Nellore. Life member of ISOI and motivated towards research in instrumentation and industrial robotics.

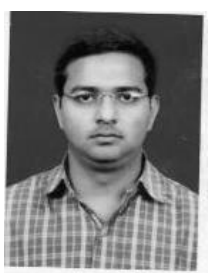

M.E.C.VIDYA SAGAR received the M.E; degree in Electronics \& Control Engineering from Sathyabama University in 2011.Currently,he is working as Senior Assistant professor in the Department of Electronics \& Instrumentation Engineering at Narayana Engineering College, Nellore. Life member of ISOI.

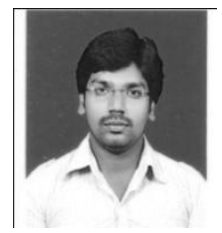

SK.NOWSHAD received the M.Tech; degree in Digital Systems and Computer Electronics Engineering from Quba College of Engineering, Nellore. Currently, he is working as Senior Assistant professor in the Department of Electronics \& Instrumentation Engineering at Narayana Engineering College, Nellore. Life member of ISOI. 